\title{
Passive Loss Inference in Wireless Sensor Networks Using EM Algorithm*
}

\author{
Yu Yang ${ }^{1,2}$, Zhulin An ${ }^{1,2}$, Yongjun $\mathrm{Xu}^{1}$, Xiaowei $\mathrm{Li}^{1}$, Canfeng $\mathrm{Chen}^{3}$ \\ ${ }^{1}$ Institute of Computing Technology, Chinese Academy of Sciences, Beijing, China \\ ${ }^{2}$ Graduate University of Chinese Academy of Sciences, Beijing, China \\ ${ }^{3}$ Nokia Research Center, Beijing, China \\ E-mail: \{yuyang, anzhulin,xyj, lxw\}@ict.ac.cn, canfeng-david.chen@nokia.com \\ Received April 20, 2010; revised May 4, 2010; accepted May 20, 2010
}

\begin{abstract}
Wireless Sensor Networks (WSNs) are mainly deployed for data acquisition, thus, the network performance can be passively measured by exploiting whether application data from various sensor nodes reach the sink. In this paper, therefore, we take into account the unique data aggregation communication paradigm of WSNs and model the problem of link loss rates inference as a Maximum-Likelihood Estimation problem. And we propose an inference algorithm based on the standard Expectation-Maximization (EM) techniques. Our algorithm is applicable not only to periodic data collection scenarios but to event detection scenarios. Finally, we validate the algorithm through simulations and it exhibits good performance and scalability.
\end{abstract}

Keywords: Wireless Sensor Networks, Passive Measurement, Network Tomography, Data Aggregation, EM Algorithm

\section{Introduction}

Recent deployments of Wireless Sensor Networks (WS$\mathrm{Ns}$ ) indicate that energy-efficient mechanisms of performance measurement are needed. The unattended nature and complexity of WSNs require that the network managers are given updated indications on the network health, for instance, the state of network links and nodes, after deployment [1]. Such information can provide early warnings of system failures, help in incremental deployment of nodes, or tuning network algorithms [1-3]. However, WSNs measurement is not a trivial task because sensor nodes have limited resources (bandwidth and power). Considering further the distributed nature of the algorithm, the large number of the nodes, and the interaction of the nodes with the environment, leads to the fact that WSNs are very hard to measure. The most commonly used approach for evaluating network performance is active measurement, which collects statistics from internal nodes directly or even injects probe messages into the network to aid in performance evaluation. However, it is usually impractical to rely on the use of active measurement in WSNs, which is not scalable or

*Supported by the National High Technology Research and Development Program of China (No. 2007AA12Z321) and the National Natural Science Foundation of China (No. 60772070; 60873244). bandwidth-efficient. On the other hand, WSNs are mainly deployed for data acquisition, thus the network performance can be passively measured by exploiting whether application data from various sensor nodes reach the sink.

The typical mode of communication in WSNs is from multiple data sources to one sink, rather than communication between any pair of nodes. Thus it usually involves tree-based topology rooted at the sink. Furthermore, since the data being collected by multiple nodes are based on common phenomena, there is likely to be some redundancy in the data being communicated. Moreover, for many node designs, the wireless transceiver requires the largest share of the overall power budget. Thus data aggregation has been put forward as a particularly useful communication paradigm for WSNs [4]. The idea of data aggregation is to combine the data coming from different sources, to eliminate redundancy, to minimize the number of transmissions and thus to save energy. In the process of data aggregation, sensor nodes in the network attempts to forward the sensing data they have collected back to the sink via a data aggregation tree. When an intermediate node in the aggregation tree receives data from multiple source nodes, it checks the contents of incoming data, combines them by eliminating redundant information and then forwards the aggregated packet to 
its parent $[1,4]$.

Specifically, WSNs can be used advantageously for periodic data collection or events detection scenarios [5]. Periodic data collection is required for operations such as tracking of the material flows, health monitoring of equipment/process. In these scenarios, all the nodes in the network are typically organized into one aggregation tree, through which nodes periodically send application data to the sink. On the other hand, WSNs are more often used in events detection scenarios, where sensor nodes are deployed to detect and classify rare and random events, such as alarm and fault detection notifications. Figure 1 depicts an example of data aggregation communication paradigm in events detection scenarios. In this example, two interested events happened in the deployment area. Then sensor nodes within a distance $\mathrm{S}$ (called the event range) and other related nodes construct two aggregation trees, through which the information of the two events collected by the sensor nodes is aggregated and sent to the sink.

In view of the unique data aggregation communication paradigm of WSNs, this study is the first to develop the network model to represent the network topology composed of multiple aggregation trees. Based on the network model, we further model the problem of link loss rates inference as a Maximum-Likelihood Estimation (MLE) problem and an inference algorithm using the standard Expectation-Maximization (EM) techniques [6] is proposed. Compared with those in wired communication networks, links in WSNs are prone to suffer high packet loss rates, which will result in unreliable and incomplete data [1]. It would thus be useful to identify links with high loss rates (lossy links) at run-time. The presented algorithm passively monitors the application traffic between sensor nodes and the sink, and then uses network tomography technology $[7,8]$ to locate lossy links. Our algorithm handles well topology changes and hence is

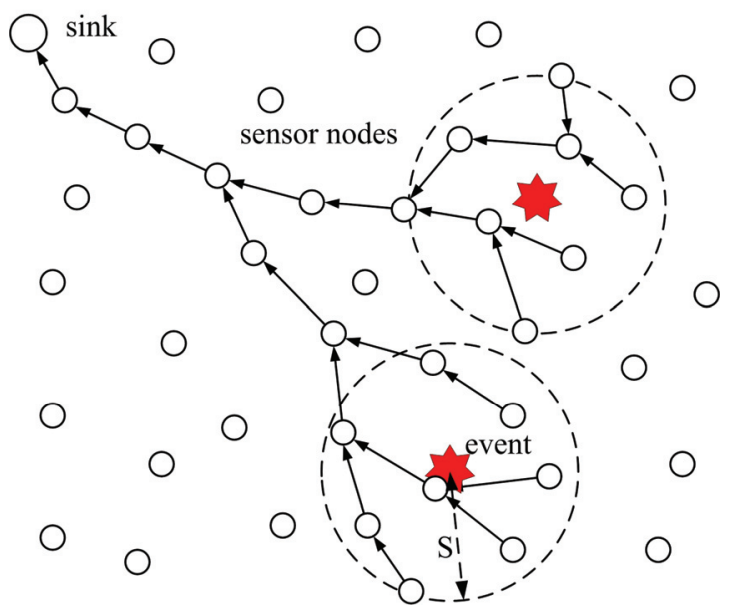

Figure 1. Data aggregation communication paradigm in events detection scenarios: An example. applicable not only to periodic data collection scenarios but to events detection scenarios.

The rest of this paper is organized as follows. Section 2 focuses on the related work. The inference models are presented in Section 3 and the inference algorithm is proposed in Section 4 based on the models. Then the simulation is shown in Section 5. Finally, Section 6 concludes the paper.

\section{Related Work}

WSNs measurement recently has received considerable attention from the research community. Existing work can be classified into two categories: active and passive measurement. Active measurement researches mainly rely on proactive approaches [9-13]. The basic idea underlying these approaches is that sensor nodes periodically report the internal status information of themselves to the sink, such as residual energy, node failures, link status, neighbor list, and the like. However, regularly sending status information from sensor nodes to the sink requires significant communication overheads, which would inevitably speed up the depletion of energy. Furthermore, contrary to wired networks where network measurement information can be delivered reliably, reports sent from sensor nodes also suffer losses. The sink has therefore no guarantee that it will receive up-to-date information.

Recently, some researches have shown that application data that sensor nodes send to the sink can be used to measure the network itself. In 2004, G. Hartl et al. considered firstly applying network tomography in WSNs based on data aggregation paradigm [14]. They formulated loss inference as an MLE problem and presented an inference algorithm based on the standard EM algorithm. However, their approach requires two restrictive assumptions: first, there is only one aggregation tree in the network and second, the aggregation tree remains static for the entire loss rate inference process. Obviously, these assumptions limit the potential applications of their algorithm in practice.

In 2005, Mao et al. employed the factor graph approach to solve the link loss inference problem [15]. In 2007, Li et al. formulated the problem of link loss rate estimation as a Bayesian inference problem and a Gibbs sampling algorithm was proposed [16]. In 2008, E. Shakshuki et al. presented an inference mechanism adopting iterative computation under Markov Chain [17]. However, these inference methods also rely on the above two assumptions. On the other hand, there are also some other researches on the loss inference problem in WSNs [18-20]. However, these approaches can only used in the situation where sensor nodes report application data to the sink through multi-hop paths separately. Thus they can not be applied to data aggregation paradigm. 


\section{Inference Models}

In this section, we develop the models which are the basis for the inference. We first model the network and then formulate the loss performance.

\subsection{Network Model}

Let $N=(V(N), L(N))$ denote a network with sets of nodes $V(N)$ and the set of links $L(N)$. The sink is denoted by $s$ and is assumed to have greater resources available than the other nodes. $(i, j) \in L(N)$ denotes a directed link from node $i$ to node $j$ in the network. Let $\Psi$ denote a set of aggregation trees embedded in $N$, i.e., $\forall T \in \Psi, V(T) \subseteq$ $V(N)$ and $L(T) \subseteq L(N)$. Note that $(i, j)$ can appear in more than one tree. Thus for $\forall(i, j) \in L(N)$, we denote $\Psi_{i, j} \subseteq \Psi$ the set of trees which include link $(i, j)$. Let $(q \rightarrow p)_{T}$ denote the path from node $q$ to node $p$ in $T$ and $L((q \rightarrow$ $p)_{T}$ ) denote the set of links on the path. The set of children of node $i$ in $T$ is denoted by $d(i, T)=\{k \in V(T) \mid(k$, i) $\in L(T)\}$. Define the set of leaf nodes in $T$ by $R(T)$, i.e., $R(T)=\{k \in V(T) \mid d(k, T)=\varnothing\}$. For $\forall k \in V(T) \backslash R(T)$, let $T(k)$ denote the subtree within $T$ rooted at $k$. Then $R(k, T)$ $=R(T) \cap V(T(k))$ is defined as the set of leaf nodes which are descended from $k$ in $T$. Also, we denote the set of all of the leaf nodes in the network by $R=\bigcup_{T \in \Psi} R(T)$.

\subsection{Loss Model}

For each link an independent Bernoulli loss process is assumed, which is a reasonable assumption [21]. Thus $\forall(i, j) \in L(N)$ can be associated with a transmission rate $\alpha_{i, j} \in(0,1)$. Let $\alpha_{i, j}$ be the probability that packets sent from node $i$ to node $j$ are received successfully by $j$. Accordingly, the loss rate of $(i, j)$ is $\bar{\alpha}_{i, j}=1-\alpha_{i, j}$. The flow of the data through an aggregation tree $T$ therefore can be modeled by a stochastic process $X_{T}=\left(X_{p, q, T}\right)_{p, q \in V(T)}$, where $X_{p, q, T} \in\{0,1\} . X_{p, q, T}=1$ means that the data sent from node $q$ were successfully received by intermediate node $p$ in the path $(q \rightarrow s)_{T}$. Similarly, $X_{p, q, T}=0$ means that the data sent from $q$ did not successfully reach $p$. Let $\alpha=\left(\alpha_{i, j}\right)_{(i, j) \in L(N)}$ denote the set of transmission rates for all links.

We assume that information about which leaf nodes' data is present in the aggregated data must also be bundled into the aggregated packet. Thus for $\forall T \in \Psi$, consider the collection of data by the sink to be an experiment. Each round of data collection will be considered a trial within this experiment. Suppose each leaf node tries to send data in each round. The outcome of each trial will be a record of which leaf nodes the sink received data from in that round. It is worth noting that the inference method in [14] needs the record about all nodes in the network, including leaf nodes and intermediate nodes. Therefore our method is more practical than that in [14].

In terms of the stochastic process $X_{T}$ defined above, each trial outcome is a random value $X_{R(T)}=\left(X_{s, k, T}\right)_{k \in R(T)}$ $\in \Omega_{R(T)}=\{0,1\}^{|R(T)|}$. Thus the overall outcome of $\Psi$ can be denoted by $X_{R}=\cup_{T \in \Psi} X_{R(T)}$. Let $\Omega_{R(T)}(i)$ be the set of outcomes $X_{R(T)}$ in which there is at least one node in $R(i, T)$ whose data were successfully received by the sink, i.e.,

$$
\Omega_{R(T)}(i)=\left\{x_{R(T)} \in \Omega_{R(T)}: \vee_{k \in R(i, T)} X_{s, k, T}=1\right\} .
$$

Let $n_{T}$ denote the data collection rounds of $T$. The probability of the $n_{T}$ independent observations of $X_{R(T)}$ is then

$$
p\left(X_{R(T)} ; \alpha\right)=\prod_{m=1}^{n_{T}} p\left(x_{R(T)}^{m} ; \alpha\right)
$$

and the probability of the observations of $X_{R}$ is

$$
p\left(X_{R} ; \alpha\right)=\prod_{T \in \Psi} p\left(X_{R(T)} ; \alpha\right) .
$$

$p\left(X_{R} ; \alpha\right)$ is the likelihood function, that is, the probability that we observe the data $X_{R}$ given the link transmission rates $\alpha$. Therefore the problem of inferring link loss rates from measurements at the sink can be formulated as an MLE problem. That is, our goal is to estimate $\alpha$ by $\hat{\alpha}$ such that $\hat{\alpha}$ maximizes the likelihood of observing the outcomes of $X_{R}$, i.e.,

$$
\hat{\alpha}=\underset{\alpha}{\arg \max } p\left(X_{R} ; \alpha\right) .
$$

However, $X_{R}$ is not sufficient to obtain a direct expression for $p\left(X_{R} ; \alpha\right)$ and then the above equation can not be solved directly. Fortunately, the standard EM techniques can be applied to the data taken from all of the trees to efficiently solve the problem. The basic idea is that rather than performing a complicated maximization, we "augment" the observable data with unobservable data so that the resulting likelihood has a simpler form.

\section{Inference Algorithm}

In this section, we further formulate the loss inference problem as an MLE problem with complete observations and then efficiently solve it using the EM algorithm. We begin by presenting some brief background information and then apply the EM algorithm to the loss inference.

\subsection{Background}

The EM Algorithm is a general method for finding MLE of parameters in statistical models, where the model depends on missing data [6]. Although a problem at first sight may not appear to be an incomplete-data one (as it is in our case), there may be much to be gained computation-wise by artificially formulating it as such to facilitate MLE. For many statistical problems, the complete- 
data likelihood has a nice form.

To be more specific, the EM algorithm attempts to find an estimate, $\hat{\alpha}$, of parameter $\alpha$ from the complete data $X_{c}$, i.e.,

$$
\hat{\alpha}=\underset{\alpha}{\arg \max } p\left(X_{c} ; \alpha\right) .
$$

Beginning with an arbitrary initial assignment, $\alpha^{(0)}$, the EM algorithm is iterative and alternates until convergence. On each iteration, there are two steps - called the Expectation (E) step and the Maximization (M) step. Hence the name EM algorithm is given to this class of techniques. The E-Step computes the conditional expected value of the complete data likelihood given the observed data, under the probability law induced by the current estimates of $\alpha$, i.e.,

$$
Q\left(\alpha \mid \hat{\alpha}^{(t)}\right)=\mathrm{E}_{\hat{\alpha}^{(t)}}\left[p\left(X_{c} ; \alpha\right) \mid X_{o b s}\right]
$$

where $\hat{\alpha}^{(t)}$ is the current estimates of $\alpha$ and $X_{o b s}$ is the observable data. In the M-Step, the expected complete data likelihood function is maximized with respect to $\alpha$ to obtain the new estimates, i.e.,

$$
\hat{\alpha}^{(t+1)}=\underset{\alpha}{\arg \max } Q\left(\alpha \mid \hat{\alpha}^{(t)}\right) .
$$

\subsection{Application to Loss Inference}

In our loss inference problem, the observable data, $X_{o b s}$, is $X_{R}$. Following the method in the above section, we augment the actual observations with the unobservable observations at the interior nodes. To be more specific, for $\forall T \in \Psi$, it is assumed that for $\forall(i, j) \in L(T)$, we can observe at node $j$ whether the packets sent from node $i$ successfully reach $j$ in every round of data collection. In terms of the stochastic process $X_{T}$ defined in Subsection 3.2 , the complete data of $T$ are $X_{c(T)}=\left(X_{j, i, T}\right)_{(i, j) \in L(T)}$. Base on the independent Bernoulli distribution assumption in Subsection 3.2, therefore, it is easy to realize that the likelihood function of $X_{c(T)}$ can be written as

$$
p\left(X_{c(T)} ; \alpha\right)=\prod_{(i, j) \in L(T)} \alpha_{i, j}^{n_{j, i, T}} \cdot \bar{\alpha}_{i, j}^{n_{T}-n_{j, i, T}},
$$

where $n_{j, i, T}$ is the number of those outcomes of $X_{j, i, T}$ whose value is equal to 1 . Similarly to (3), the likelihood function of the complete data of $\Psi, X_{c}=\left(X_{c(T)}\right)_{T \in \Psi}$, is

$$
p\left(X_{c} ; \alpha\right)=\prod_{T \in \Psi} p\left(X_{c(T)} ; \alpha\right) \text {. }
$$

It is convenient to work with the log-likelihood function, $\mathcal{L}\left(X_{c} ; \alpha\right)=\log p\left(X_{c} ; \alpha\right)$, which is given by

$$
\begin{aligned}
\mathcal{L}\left(X_{c} ; \alpha\right)= & \sum_{(i, j) \in L(N)}\left(\sum_{T \in \Psi_{i, j}} n_{j, i, T} \log \alpha_{i, j}\right. \\
& \left.+\left(\sum_{T \in \Psi_{i, j}} n_{T}-\sum_{T \in \Psi_{i, j}} n_{j, i, T}\right) \log \bar{\alpha}_{i, j}\right) .
\end{aligned}
$$

It can be seen from Subsection 4.1 that the key of the EM algorithm is to compute the conditional expectation of the complete data likelihood. Based on (6) and (10), we can obtain

$$
\begin{aligned}
Q\left(\alpha \mid \hat{\alpha}^{(t)}\right)= & \mathrm{E}_{\hat{\alpha}^{(t)}}\left[\mathcal{L}\left(X_{c} ; \alpha\right) \mid X_{R}\right] \\
= & \sum_{(i, j) \in L(N)}\left(\sum_{T \in \Psi_{i, j}} \hat{n}_{j, i, T} \log \alpha_{i, j}\right. \\
& \left.+\left(\sum_{T \in \Psi_{i, j}} n_{T}-\sum_{T \in \Psi_{i, j}} \hat{n}_{j, i, T}\right) \log \bar{\alpha}_{i, j}\right),
\end{aligned}
$$

where $\hat{n}_{j, i, T}$ is the conditional expectation of $n_{j, i, T}$ given the observable data $X_{R(T)}$ under the probability law induced by $\hat{\alpha}^{(t)}$, i.e.,

$$
\hat{n}_{j, i, T}=\mathrm{E}_{\hat{\alpha}^{(t)}}\left[n_{j, i, T} \mid X_{R(T)}\right] .
$$

Remember that

$$
n_{j, i, T}=\sum_{m=1}^{n_{T}} x_{j, i, T}^{m}
$$

Thus we have

$$
\begin{aligned}
\hat{n}_{j, i, T}= & \sum_{m=1}^{n_{T}} \mathrm{P}_{\hat{\alpha}^{(t)}}\left[X_{j, i, T}=1 \mid X_{R(T)}=x_{R(T)}^{m}\right] \\
= & \sum_{x_{R(T)} \in \Omega_{R(T)}} n_{T}\left(x_{R(T)}\right) \mathrm{P}_{\hat{\alpha}^{(t)}} \\
& {\left[X_{j, i, T}=1 \mid X_{R(T)}=x_{R(T)}\right], }
\end{aligned}
$$

where $n_{T}\left(x_{R(T)}\right)$ denotes the number of collection rounds for which the outcome $x_{R(T)}$ is obtained. Then the problem is turned into the computation of the conditional probability, $\mathrm{P}_{\hat{\alpha}^{(t)}}\left[X_{j, i, T}=1 \mid X_{R(T)}=x_{R(T)}\right]$. To facilitate the computation, we divide the set of outcomes $x_{R(T)}$ into two groups:

1) $x_{R(T)} \in \Omega_{R(T)}(i)$

This group of outcomes implies that there is at least one node in $R(i, T)$ whose data are aggregated at node $i$ and then successfully received by the sink through path $(i$ $\rightarrow s)_{T}$. This clearly indicates that node $j$ successfully received data from node $i$, and hence

$$
\mathrm{P}_{\hat{\alpha}^{(t)}}\left[X_{j, i, T}=1 \mid X_{R(T)}=x_{R(T)}, x_{R(T)} \in \Omega_{R(T)}(i)\right]=1 \text {. }
$$

2) $x_{R(T)} \in \Omega_{R(T)} \backslash \Omega_{R(T)}(i)$

This group of outcomes suggests that there is none of nodes in $R(i, T)$ whose data successfully reached the sink. Obviously, this does not indicate whether node $j$ successfully received data from node $i$. Thus we divide this group of outcomes into two categories and handle them accordingly (for clarity, let $X_{\bar{\Omega}}$ denote the situation $\left.\left\{X_{j, i, T}=1 \mid X_{R(T)}=x_{R(T)}, x_{R(T)} \in \Omega_{R(T)} \backslash \Omega_{R(T)}(i)\right\}\right)$ :

a. There is at least one node in $R(i, T)$ whose data reach node $i$, i.e., $\vee_{k \in R(i, T)} X_{i, k, T}=1$. In this case, the data should reach node $j$ and then be lost on path $(j \rightarrow s)_{T}$. Thus, 


$$
\begin{aligned}
& \mathrm{P}_{\hat{\alpha}^{(t)}}\left[X_{\bar{\Omega}} \mid \vee_{k \in R(i, T)} X_{i, k, T}=1\right] \\
& =\hat{\alpha}_{i, j}^{(t)} \cdot\left(1-\prod_{(p, q) \in L\left((j \rightarrow s)_{T}\right)} \hat{\alpha}_{p, q}^{(t)}\right) .
\end{aligned}
$$

b. There is none of nodes in $R(i, T)$ whose data reach node $i$, i.e., $\vee_{k \in R(i, T)} X_{i, k, T}=0$. In this case, the value of $X_{j, i, T}$ is independent of $X_{R(T)}$ and depends only on the performance of link $(i, j)$. Thus

$$
\mathrm{P}_{\hat{\alpha}^{(t)}}\left[X_{\bar{\Omega}} \mid \vee_{k \in R(i, T)} X_{i, k, T}=0\right]=\hat{\alpha}_{i, j}^{(t)}
$$

For $\forall i \in V(T) \backslash R(T)$, define $\beta(i, T)$ to be the probability that there is at least one node in $R(i, T)$ whose data reach node i, i.e.,

$$
\beta(i, T)=\mathrm{P}_{\hat{\alpha}^{(t)}}\left[\vee_{k \in R(i, T)} X_{i, k, T}=1\right] .
$$

Based on the formula of total probability, therefore, for $x_{R(T)} \in \Omega_{R(T)} \backslash \Omega_{R(T)}(i)$, we obtain

$$
\begin{aligned}
\mathrm{P}_{\hat{\alpha}^{(t)}}\left[X_{\bar{\Omega}}\right] & =\mathrm{P}_{\hat{\alpha}^{(t)}}\left[X_{\bar{\Omega}} \mid \underset{k \in R(i, T)}{\vee} X_{i, k, T}=1\right] \cdot \beta(i, T) \\
& +\mathrm{P}_{\hat{\alpha}^{(t)}}\left[X_{\bar{\Omega}} \mid \underset{k \in R(i, T)}{\vee} X_{i, k, T}=0\right] \cdot \bar{\beta}(i, T) .
\end{aligned}
$$

$\bar{\beta}(i, T)=1-\beta(i, T)$ is the probability that $i$ does not receive data from any node in $R(i, T)$. This means that $i$ does not receive data from any leaf node descended from all of $i$ 's children, namely from $R(q, T), q \in d(i, T)$. For any $R(q)$, there are two reasons that $i$ does not receive data from it, one is that $q$ does not receive data from any node in $R(q)$, the other is that $q$ receives data from $R(q)$, but the data are lost on link $(q, i)$. Thus for $\forall i \in V(T) \backslash$ $R(T)$, the following recursive equation can be obtained,

$$
\bar{\beta}(i, T)=\prod_{q \in d(i, T)}\left(\bar{\beta}(q, T)+\beta(q, T) \cdot\left(1-\hat{\alpha}_{q, i}^{(t)}\right)\right) .
$$

Using (20) we can recursively compute $\beta(i, T)$ for $\forall i \in$ $V(T)$ based on $\hat{\alpha}^{(t)} \quad($ for $\forall i \in R(T), \beta(i, T)=1$ ).

While combining(14), (15), and (19), we can work out $\hat{n}_{j, i, T}$ and then use (11) to compute the conditional expectation of the complete data likelihood, $Q\left(\alpha \mid \hat{\alpha}^{(t)}\right)$.

For the M-Step of the EM algorithm, maximization of $Q\left(\alpha \mid \hat{\alpha}^{(t)}\right)$ is trivial, as the stationary point conditions

$$
\frac{\partial Q\left(\alpha \mid \hat{\alpha}^{(t)}\right)}{\partial \alpha_{i, j}}=0, \quad(i, j) \in L(N) .
$$

immediately yield

$$
\hat{\alpha}_{i, j}^{(t+1)}=\frac{\sum_{T \in \Psi_{i, j}} \hat{n}_{j, i, T}}{\sum_{T \in \Psi_{i, j}} n_{T}}, \quad(i, j) \in L(N) .
$$

\subsection{Algorithm Description}

The algorithm starts with an arbitrary initial assignment of link transmission rates, $\hat{\alpha}^{(0)}$. Simulations suggest that the values that the algorithm converges to are independent of initial values, which is a property of the EM algorithm. Then the E-step and M-step are iterated until the inferred transmission rate of each link changes by less than a termination criterion between consecutive iterations. Finally, the algorithm use a threshold $t_{l}$ to decide whether a link is lossy. That is, for $\forall(i, j) \in L(N)$, if $\hat{\alpha}_{i, j}$ lies below $t_{l},(i, j)$ is added to the solution set of lossy links, $\mathcal{X}$, which originally is empty. The algorithm is presented in Table 1.

\section{Simulation}

\subsection{Simulation Setup}

The algorithm is simulated over OMNeT++ network simulator. Random networks consisting of 500, 1000 and 2000 nodes (N500, N1000 and N2000) are generated. Furthermore, two different distributions are used for assigning loss rates to links. The first loss distribution (LD1) is a random selection model, where the intended link loss rate is selected randomly in the interval $[0.01,0.4]$. In the second loss distribution (LD2), link transmission rates are drawn from a distribution with probability density function $f(\alpha)=\lambda \alpha^{\lambda-1}$, for $0<\alpha<1$ parameterized by $\lambda>$ 1. The expected value of this random variable is $\lambda /(1+$ $\lambda$ ). In our simulations, $\lambda$ is chosen as 4 so that the expected link loss rate is 0.2 .

\section{Table 1. The description of the EM algorithm.}

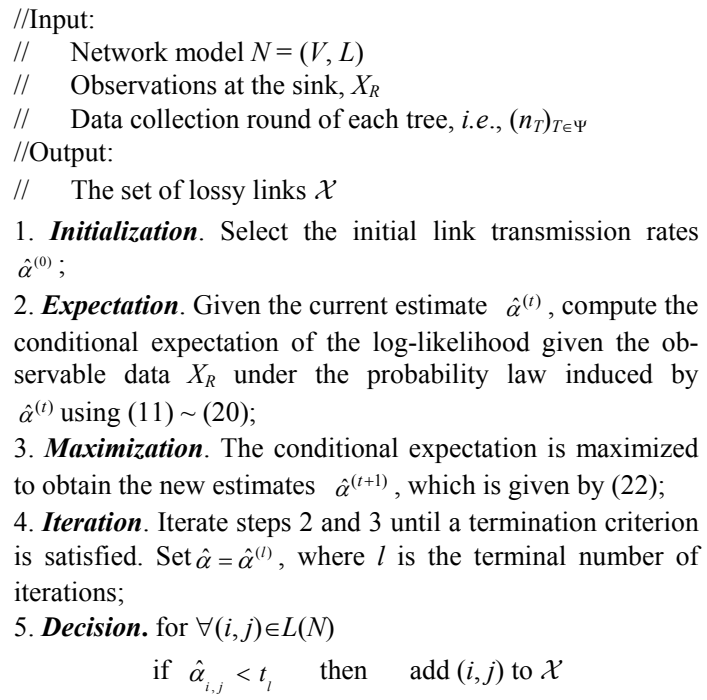


We say a link is lossy if its transmission rate lies below 0.8 (i.e., its loss rate is above 0.2); otherwise, it is normal. The termination criterion of the algorithm is chosen as 0.0001. Moreover, the performance of the algorithm is evaluated using two metrics: the detection rate (DR), which is the percentage of links that are correctly inferred as lossy, and the false positive rate (FPR), which is the percentage of links that are normal but are inferred as lossy. With $\mathcal{F}$ denoting the set of the actual lossy links and $\mathcal{X}$ the set of links identified as lossy by the algorithm, these two rates are given by:

$$
\mathrm{DR}=|\mathcal{F} \cap \mathcal{X}| / \mathcal{F} ; \mathrm{FPR}=|\mathcal{X} \backslash \mathcal{F}| / \mathcal{X} .
$$

\subsection{Simulations in Periodic Data Collection Scenarios}

The algorithm is first evaluated in periodic data collection scenarios. In this group of simulations, one random tree topology is generated in every network. The branching ratio at each non-leaf node is randomly chosen between 1 and an upper bound of 10. The data collection rounds in each simulation are chosen as 200 and the algorithm is performed every 20 rounds to observe its convergent tendency.

Figure 2 and Figure 3 plot DR and FPR of the algorithm with LD1 and LD2 respectively. We observe that the algorithm is quite insensitive to different loss distributions. We also note that the algorithm shows good scalability. As the network size increases, the algorithm has high DR and low FPR and the accuracy slightly decreases. Furthermore, we observe the accuracy increases as the data collection rounds increases and the algorithm converges fast: when the data collection rounds reach $100, \mathrm{DR}$ is about 0.9 and FPR is about 0.1 .

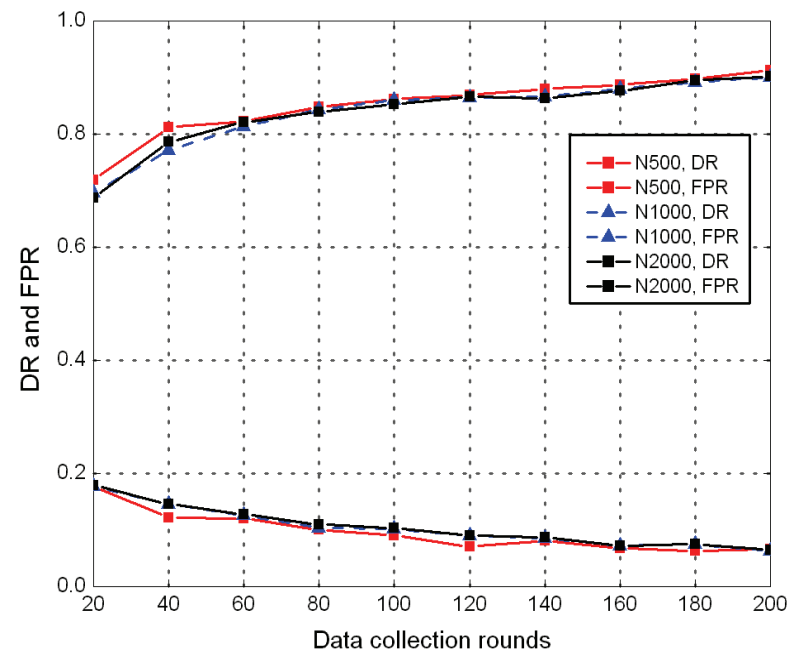

Figure 2. DR and FPR with LD1.
Figure 4 shows how accurate the inference is when the links are rank ordered based on our "confidence" in the inference. We quantify the confidence as the inferred loss rates of the links. The links in N1000 with LD2 are considered in the order of decreasing inferred values (the data collection rounds are 100). We plot 3 curves: the true number of lossy links in the set of links considered up to that point, the number of correct inferences, and the number of false positives. We note that the confidence rating assigned by the algorithm works very well. There are zero false positives for the top 112 rank ordered links. Moreover, each of the first 346 true lossy links in the rank ordered list is correctly identified as lossy (i.e., none of these true lossy links is "missed"). These results suggest that the confidence estimate of the algorithm can be used to rank the order of the inferred lossy links so that the top few inferences are (almost) perfectly accurate. This is likely to be useful in a practical setting where we may want to identify at least a small number of lossy links with certainty so that corrective action can be taken.

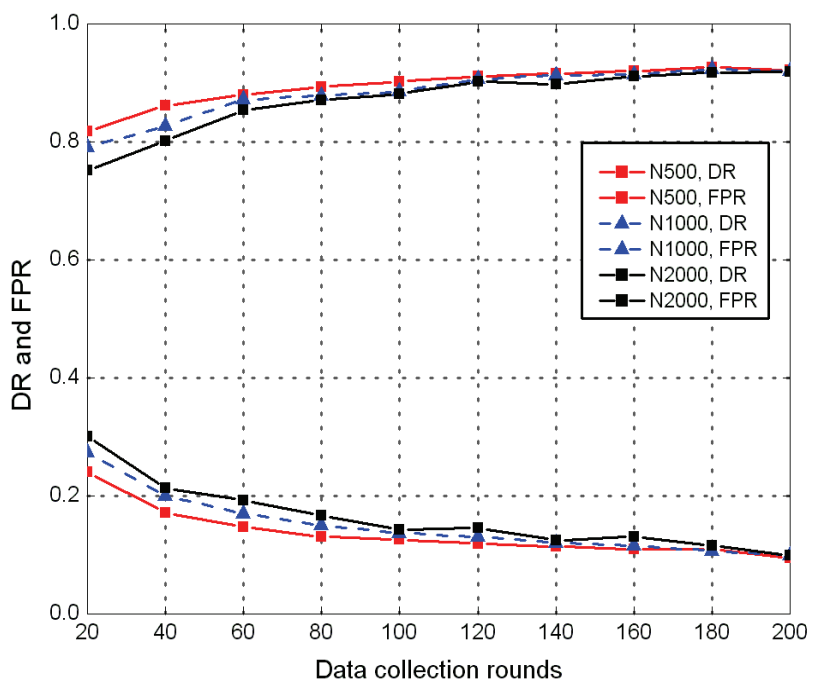

Figure 3. DR and FPR with LD2.

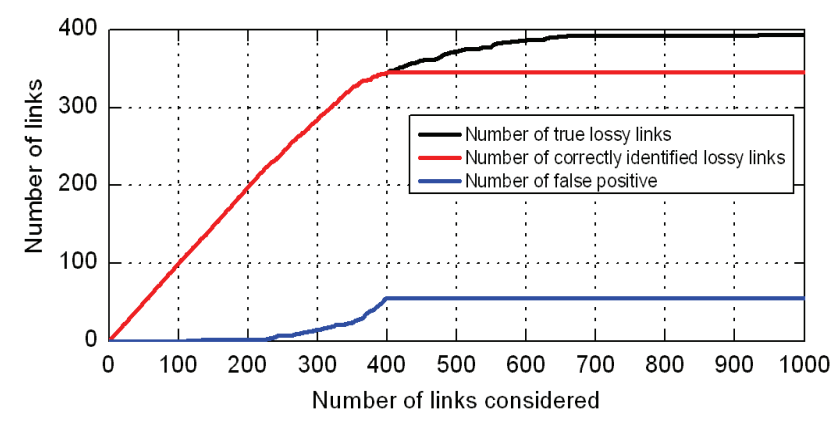

Figure 4. The links are rank ordered based on inference confidence (N1000, LD2, 100 collection rounds). 


\subsection{Simulations in Events Detection Scenarios}

In this section, the algorithm is evaluated in events detection scenarios. The experimental setup of this group of simulations is as follows. The networks are randomly placed in a square area of D size and the sink is located at the upper left corner of the area. The communication radius of sensor nodes is set as $0.05 \mathrm{D}$. In each experiment, 100 events randomly happen one after another in the area with the event range $\mathrm{S}$. For each event, a data aggregation tree is constructed using the greedy incremental tree algorithm proposed in [22] to transmit the event information to the sink. See Figure 1. After data collection, the algorithm is performed on the observations of all trees to obtain the combined results.

The simulations are divided into two groups. The first group of simulations is used to evaluate the convergence of the algorithm in events detection scenarios. In this group the data collection rounds of each aggregation tree are selected randomly in $[0,50],[50,100]$ or $[100,150]$ and the event ranges are set as $0.1 \mathrm{D}$. The second group is used to investigate the performance of our algorithm with different scales of aggregation trees in events detection scenarios. In this group the event ranges vary between $0.05 \mathrm{D}, 0.1 \mathrm{D}$ and $0.15 \mathrm{D}$ while the data collection rounds of each aggregation tree are uniformly distributed in $[50,100]$.

Figure 5 and Figure 6 depict the simulation results of the first group of experiments. The figures suggest that the algorithm is also robust against different loss distributions and scales well. Furthermore, the algorithm shows good convergence: even through the collection rounds of each tree are less than 50, the algorithm still has high DR and low FPR. The underlying reason for this success can be attributed to the fact that for the links that are contained by more than one trees, the algorithm can combine more observations and therefore the results are more accurate.

The simulation results of the second group of experiments are illustrated in Figure 7 and Figure 8. We also note that the algorithm performs similarly under different

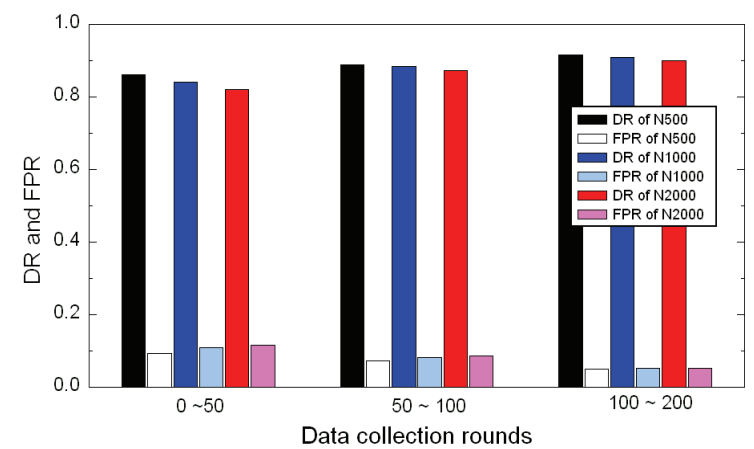

Figure 5. DR and FPR with different data collection rounds of each aggregation tree (LD1).

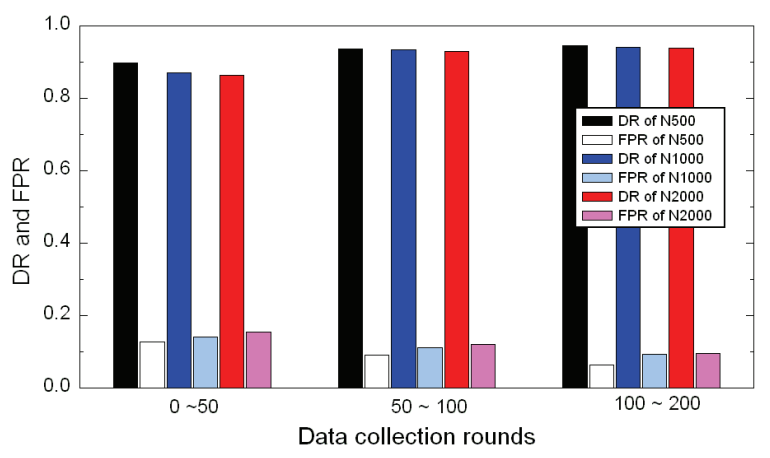

Figure 6. DR and FPR with different data collection rounds of each aggregation tree (LD2).

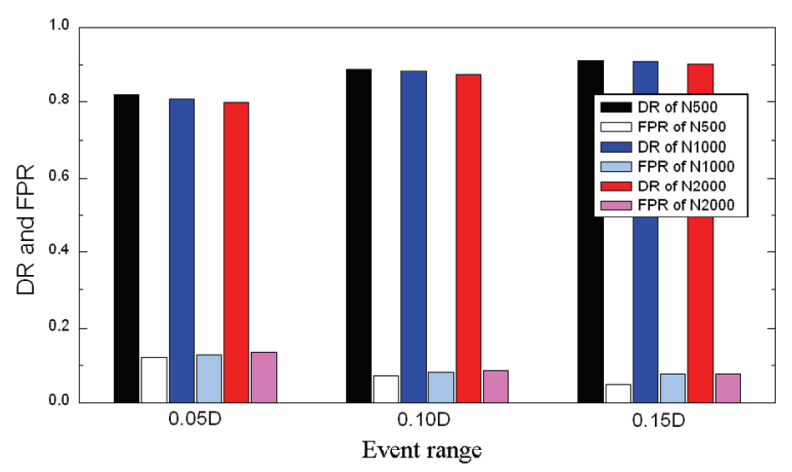

Figure 7. DR and FPR with different event ranges (LD1).

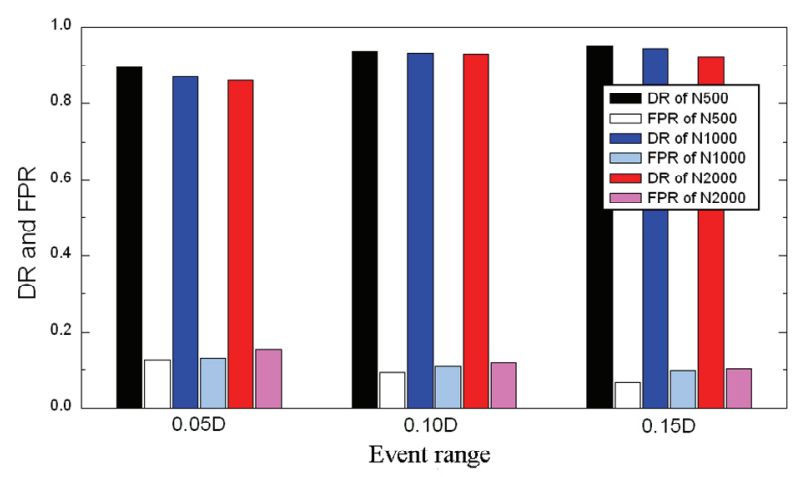

Figure 8. DR and FPR with different event ranges (LD2).

network scales and loss distributions. Moreover, we observe that its accuracy increases as evnt range, $\mathrm{S}$, increases. The number of the links that shared by multiple trees will increase when $\mathrm{S}$ increase. Thus the algorithm can perform more accurately by combining more observations.

\section{Conclusions}

In this paper we consider the problem of inferring link loss rates from the application traffic between sensor nodes and the sink taken from a collection of aggregation 
trees. Based on the data aggregation communication paradigm, we propose a practical loss performance inference algorithm using the standard Expectation-Maximization (EM) techniques. Our algorithm simply observes the application traffic of the network and handles well topology changes. Therefore, our approach is applicable not only to periodic data collection scenarios but also to events detection scenarios. Via simulations varying between different network scales, application scenarios, loss distributions, it can be observed that the algorithm converges fast and exhibits good scalability and stability.

\section{References}

[1] C. G. Vehbi and P. H. Gerhard, "Industrial Wireless SenSor Networks: Challenges, Design Principles, and Technical Approaches," IEEE Transactions on Industrial Electronics, Vol. 56, No. 10, October 2009, pp. 4258-4265.

[2] J. A. Stankovic, "When Sensor and Actuator Networks Cover the World," ETRI Journal, Vol. 30, No. 5, October 2008, pp. 627-633.

[3] A. Willig, "Recent and Emerging Topics in Wireless Industrial Communications: A Selection," IEEE Transactions on Industrial Informatics, Vol. 4, No. 2, May 2008, pp. 102-124.

[4] E. Fasolo, M. Rossi, J. Widmer and M. Zorzi, "In-Network Aggregation Techniques for Wireless Sensor Networks: A Survey," IEEE Wireless Communications, Vol. 14, No. 2, 2007, pp. 70-86.

[5] K. S. Low, W. N. N. Win and M. J. Er, "Wireless Sensor Networks for Industrial Environments," Proceedings of International Conference on Computational Intelligence for Modelling, Control and International Conference on Automation and Intelligent Agents, Web Technologies and Internet Commerce, Vienna, Vol. 2, 2005, pp. 271276.

[6] G. J. McLachlan and T. Krishnan, "The EM Algorithm and Extensions," 2nd Edition, John Wiley and Sons, Inc, New York, 2008.

[7] R. Castro, M. Coates, G. Liang, R. Nowak and B. Yu, "Network Tomography: Recent Developments," Statistical Science, Vol. 19, No. 3, 2004, pp. 499-517.

[8] B. Tian, D. Nick, P. Francesco Lo and T. Don, "Network Tomography on General Topologies," ACM SIGMETRICS Performance Evaluation Review, Vol. 30, No. 1, 2002, pp. 21-30.

[9] S. Rost and H. Balakrishnan, "Memento: A Health Monitoring System for Wireless Sensor Networks," Proceedings of 3rd Annual IEEE Communications Society on Sensor and Ad Hoc Communications and Networks, Santa Clara, Vol. 3, 2006, pp. 575-584.

[10] X. Meng, T. Nandagopal, L. Li and S. Lu, "Contour Maps: Monitoring and Diagnosis in Sensor Networks," Computer Networks, Vol. 50, No. 15, 2006, pp. 28202838 .
[11] N. Ramanathan, K. Chang, R. Kapur, L. Girod and E. Kohler, "Sympathy for the Sensor Network Debugger," Proceedings of ACM Conference on Embedded Networked Sensor Systems (SenSys), San Diego, 2005, pp. 255-267.

[12] S. Gupta, R. Zheng and A. M. K. Cheng, "ANDES: An Anomaly Detection System for Wireless Sensor Networks," Proceedings of IEEE International Conference on Mobile Adhoc and Sensor Systems (MASS), Pisa, 2007, pp. 1-9.

[13] A. Meier, M. Motani, S. Hu and K. Simon, "DiMo: Distributed Node Monitoring in Wireless Sensor Networks," Proceedings of International Symposium on Modeling, Analysis and Simulation of Wireless and Mobile Systems, Vancouver, 2008, pp. 117-121.

[14] G. Hartl and B. Li, "Loss Inference in Wireless Sensor Networks Based on Data Aggregation," Proceedings of International Symposium on Information Processing in Sensor Networks (IPSN), Berkeley, 2004, pp. 396-404.

[15] Y. Mao, F. R. Kschischang, B. Li and S. Pasupathy, "A Factor Graph Approach to Link Loss Monitoring in Wireless Sensor Networks," IEEE Journal on Selected Areas in Communications, Vol. 23, No. 4, 2005, pp. 820829.

[16] Y. Li, W. Cai, G. Tian and W. Wang, "Loss Tomography in Wireless Sensor Network Using Gibbs Sampling," Proceedings of European Conference on Wireless Sensor Networks, Delft, 2007, pp. 150-162.

[17] E. Shakshuki and X. Xing, "A Fault Inference Mechanism in Sensor Networks Using Markov Chain," Proceedings of the 22nd International Conference on $\mathrm{Ad}$ vanced Information Networking and Applications, GinoWan, 2008, pp. 628-635.

[18] H. X. Nguyen and P. Thiran, "Using End-To-End Data to Infer Lossy Links in Sensor Networks," Proceedings of IEEE International Conference on Computer Communications (INFOCOM), Barcelona, 2006, pp. 2205-2216.

[19] K. Liu, M. Li, Y. Liu, M. Li and Z. Guo, "Passive DiaGnosis for Wireless Sensor Networks," Proceedings of ACM Conference on Embedded Network Sensor Systems (SenSys), New York, 2008, pp. 113-126.

[20] B. Wang, W. Wei, W. Zeng and R. P. Krishna, "Fault Localization Using Passive End-to-End Measurement and Sequential Testing for Wireless Sensor Networks," Proceedings of IEEE Communications Society Conference on Sensor, Mesh and Ad Hoc Communications and Networks, Rome, 2009, pp. 225-234.

[21] A. Woo, T. Tong and D. Culler, "Taming the Underlying Challenges of Reliable Multi-Hop Routing in Sensor NetWorks," Proceedings of ACM Conference on Embedded Networked Sensor Systems, Los Angeles, 2003, pp. 1417.

[22] B. Krishnamachari, D. Estrin and S. Wicker, "The Impact of Data Aggregation in Wireless Sensor Networks," Proceedings of the 22nd International Conference on Distributed Computing Systems, Vienna, 2002, pp. 575-578. 